\section{La voix des internistes généraux canadiens}

Alors qu'une nouvelle décennie s'ouvre à nous, c'est l'occasion pour certains d'entre nous de réfléchir sur les réalisations et les étapes franchies au cours de la décennie écoulée, tandis que pour d’autres, il s'agit simplement d'un autre moment fugace dans le continuum espace-temps. Au moment où jécris, en ce jour palindromique (02022020), cet espace me donne l'occasion privilégiée de partager mes réflexions sur le Journal et, avec une certaine humilité, sur notre parcours de cliniciens dans le monde de la médecine interne générale.

Le Journal canadien de médecine interne générale - votre journal - entre dans sa troisième décennie d'existence, ayant été lancé en 2006 par le Dr Hector Baillie qui est maintenant un contributeur permanent au segment "Casebook of a Community Internist” du Journal. Plus récemment, la revue a été dirigée par le Dr Mitchell Levine, qui en a accru le profil académique et est passé à un format en ligne nous permettant d'atteindre un public plus large de manière plus rentable (et en épargnant les arbres). Alors que j’entre dans la troisième année de mon mandat de rédacteur en chef, mon objectif principal pour la décennie en cours est de faire en sorte que la Revue obtienne le statut d'index de la Bibliothèque nationale de médecine des États-Unis. Avec les rédacteurs en chef adjoints, les docteurs Rodrigo Cavalcanti, Ameen Patel et John You, nous avons entamé le processus vers cet objectif, et lorsqu'il sera atteint (et non pas s'il l'est), il servira de tremplin pour attirer la plus haute bourse d'études en médecine interne générale (MGI) au Canada et à l'étranger. Toutefois, nous conserverons toujours la mission de la Revue, qui est d'être la voix des internistes généraux canadiens.

En effet, le chœur des voix appartenant aux internistes généraux a également, au cours de la dernière décennie, connu un crescendo écrasant. Le catalyseur, à mon avis, s'est produit en 2011 lorsque le Collège royal des médecins et chirurgiens du Canada a accordé le statut de sous-spécialité à la GIM. Ce changement, associé au développement de programmes de formation en GIM dans tout le Canada, dirigé par de nombreux directeurs de programmes dévoués, a entraîné un regain d'intérêt pour la GIM, qui est devenue l'une des destinations les plus prisées des diplômés des écoles de médecine. On peut dire sans se tromper que dans la pratique médicale, les années 10 ont été la décennie de la GIM au Canada.

Enfin, au milieu de la nouvelle épidémie de coronavirus qui sévit actuellement, nous pouvons également réfléchir à nos positions privilégiées en tant qu'internistes généralistes dans un pays dont le système de santé, bien qu'imparfait, fait l'envie de nombreuses autres nations. Embrassons la nouvelle décennie avec vigueur, détermination et humilité.

James Douketis MD

Rédacteur en chef

Revue canadienne de médecine interne générale

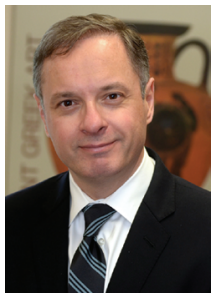

